\title{
O Acompanhamento Terapêutico e a psicanálise: pequeno histórico e caso clínico*1
}

\author{
Sonia Alberti*2 \\ Leônia Cavalcante Teixeira*3 \\ Irene Moura Beteille*4 \\ Sonia Wan Der Maas Rodrigues*5 \\ Carla Renata Braga de Souza Martinez*6
}

A partir de um breve histórico de dispositivos clínicos utilizados no campo da saúde mental, discute-se a prática do Acompanhamento Terapêutico. Tal discussão se instrumentaliza da transferência, como conceito psicanalítico. Levantamos as hipóteses de que nesse trabalho realizado cotidianamente fora dos moldes do consultório, a fala de um usuário instiga a uma articulação entre teoria e prática e que a própria psicanálise pode dela adquirir um novo saber fazer clínico.

Palavras-chave: Psicanálise, dispositivo clínico, acompanhante terapêutico, caso clínico

${ }^{* 1}$ Artigo baseado em mesa-redonda no $8^{\circ}$ CONPSI - Psicologia, contemporaneidade e inserção social: desafios e perspectivas, intitulada Dispositivos clínicos para um cuidado no cotidiano: psicanálise e saúde coletiva, com trabalhos apresentados por Irene de Mouta Beteille (orientada por Sonia Alberti), Sonia Wan Der Maas Rodrigues e Carla Renata Braga de Souza Martinez (orientadas por Leônia Cavalcante Teixeira).

*2 Universidade do Estado do Rio de Janeiro - UERJ (Rio de Janeiro, RJ, Br).

*3 Universidade de Fortaleza - Unifor (Fortaleza, CE, Br).

${ }^{*}$ Doutoranda da Universidade do Estado do Rio de Janeiro - UERJ (Rio de Janeiro, $\mathrm{RJ}, \mathrm{Br})$.

${ }^{* 5,6}$ Doutorandas da Universidade de Fortaleza - Unifor (Fortaleza, CE, Br). 


\section{SAÚDE MENTAL}

A psicanálise é, desde sua gênese, construída numa imbricação com o fazer clínico ao mesmo tempo em que se apresenta como uma metodologia de pesquisa, sempre clínica, o que orienta sua ética. Rompendo com o positivismo da época, Freud inaugura um novo "paradigma cultural" quando se dispõe a "tratar seus pacientes, não investigando seus organismos, mas convidando-os a associar livremente" (Nogueira, 2004, p. 84). Por outro lado, a instância psíquica do inconsciente não pode ser estudada isoladamente, pois há sobre ela influências externas — do social, da política, da economia - que repercutem diretamente no sujeito (Elia, 2000), sendo isso ratificado a partir da escuta do que os pacientes trazem em suas falas nos diferentes moldes de atendimento, os quais adentram as instituições diversas por meio dos chamados dispositivos clínicos.

A última reforma psiquiátrica brasileira "possuiu um forte apelo social" com "argumentos fundamentados em alguns aspectos de teorias sociológicas" (Couto \& Alberti, 2008, p. 23), mas não basta garanti-la com leis e portarias. Com efeito, a cidadania dos usuários da rede de saúde mental por muito tempo ainda se submeteu ao discurso do mestre que vigia nesse campo antes de que a dita reforma pudesse, verdadeiramente, subvertê-lo. Além do mais, teorias sociológicas aplicadas ao Estado introduziram uma articulação com o discurso universitário e, portanto, com a burocracia (idem, p. 23), a ponto de ainda hoje constituir possíveis orientações. Mas há verdadeiras mudanças que se pode observar e uma delas é encetada com a prática do Acompanhamento Terapêutico, tema que pretendemos desdobrar neste trabalho.

\section{O Acompanhamento Terapêutico (AT)}

Os primeiros textos sobre o AT surgiram no Brasil a partir de 2005. Na linguagem cotidiana, conforme o Dicionário Aurélio Buarque de Holanda, o vocábulo dispositivo é um aparelho construído ou um conjunto de ações planejadas e coordenadas visando a um determinado fim. Foi Foucault (1979) quem construiu um saber em torno do que ele chama de 


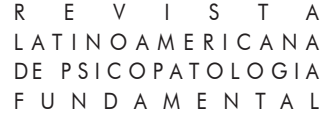

"dispositivos clínicos-institucionais", atribuindo uma função metodológica a essa expressão. Trata-se, para ele, de um conjunto heterogêneo, formado de ditos e não ditos, "rede que se pode estabelecer entre estes elementos" (p. 244), sempre inscrita num jogo de poder.

Foucault levanta uma discussão em que o dispositivo clínico-institucional, estando inserido numa relação de poder, produz subjetividades tendo em vista um corpo que é entrecortado pelos elementos normatizadores que compõem o dispositivo, corpo docilizado a partir de uma relação de poder que está para além do que é assumido em palavras. Sobre isso Agamben (2005) afirma que "certamente o termo, no uso comum como no foucaultiano, parece se referir à disposição de uma série de práticas e de mecanismos (...) com o objetivo de fazer frente a uma urgência e de obter um efeito" (p. 11). Efeito que permeia o percurso o qual, como já dizia Veloso Filho (2005), identifica a própria psicanálise com o dispositivo clínico.

Para além do que ficou conhecido como setting clínico freudiano na realidade, muitas vezes Freud atendia seus pacientes caminhando pelos parques vienenses - , a psicanálise nunca deixou de se dirigir para o social (cf. Freud, 1912/1996a; 1918/1996b; 1921/1996d; 1930/1996e), o que Lacan (2003) inscreveria numa das seções de sua Escola, como Psicanálise em Extensão. Nesse contexto, a prática psicanalítica na instituição é balizada por princípios teóricos, metodológicos, éticos e políticos, podendo contribuir, pelo menos, de três formas: com a escuta psicanalítica dos pacientes, levando em conta a especificidade da situação; como um analisador externo à instituição no modelo do mais um, tendo função de provocar efeitos de elaboração a respeito do que é discutido institucionalmente; como um dos membros da equipe formuladora e instauradora do processo institucional.

Ou, como já diziam Rinaldi e Bursztyn (2008), "entendemos como dispositivo clínico o conjunto de mecanismos, orientados a partir de um determinado campo de saber, que implicam determinadas posições neste campo e se dispõem a um determinado fim no cenário clínico" (p. 39), visando a aplicabilidade do saber da psicanálise para além do molde tradicional do setting clínico. O AT, no que tange à Psicanálise em Extensão, não é aí mais do que um modelo primordial de dispositivo clínico, e a construção do caso clínico é um eixo fundamental para fazê-lo funcionar (sobre a questão da construção do caso clínico, sugerimos a leitura de: Andrade, 2005; Borges, 2010; Bursztyn \& Figueiredo, 2012; Dunker, 2011; Figueiredo, 2004; Val \& Lima, 2014; Viganó, 1999; Vorcaro, 2010).

A pergunta que construímos é: haveriam dispositivos clínicos em saúde mental que permitiriam verificar que isso pode efetivamente acontecer no 


\section{SAÚDE MENTAL}

âmbito institucional, testemunhando que, para além do "forte apelo social", a reforma psiquiátrica dos anos 1990 efetivamente está introduzindo, também, um lugar para o sujeito? Para podermos responder a essa questão, é preciso verificar primeiro o alcance do AT na clínica da saúde mental.

\section{Clínica na Saúde Mental: o que pode um acompanhante terapêutico $\left(a t^{1}\right)$ ?}

Como uma técnica de trabalho direcionada ao auxílio no tratamento psiquiátrico, o at originou-se na década de 1950, nos movimentos da Reforma Psiquiátrica da Europa e dos Estados Unidos (Couto \& Alberti 2008). Dez anos depois, chegou à América Latina, via Argentina. Naquele momento, era denominado "amigo qualificado" - para um histórico sobre a mudança do nome, ver Araújo (2005).

O AT é um dispositivo clínico bastante presente no processo de reabilitação psicossocial, tendo como um dos seus objetivos o resgate dos vínculos sociais, cidadania e circulação dos portadores de sofrimento mental nos diferentes espaços físicos e sociais. Para Palombini (2006), acompanhar o usuário é uma forma de implementação da reforma psiquiátrica, uma vez que faz acontecer a desinstitucionalização da loucura, contribuindo para o exercício da autonomia do usuário, de forma que possa assumir, aos poucos, funções na comunidade. Daí ter se tornado uma estratégia de antissegregação e prevenção contra a alienação social (Bertolote, 1996; Pitta, 1996).

Saraceno (1998) assim descreve essa modalidade terapêutica como uma forma de artesanato da clínica no sentido que ela oferece "possibilidades para permitir ao paciente experimentar a intermitência de seu sofrimento, uma clínica que produza intercâmbio entre os pacientes, enquanto estão sofrendo, com outras pessoas" (p. 30). Essa clínica, aparentemente tão distante do modelo encontrado em consultórios, só pode se dar a partir do momento em que se leva em conta o lugar do desejo e da subjetividade. Tem objetivos, projeto terapêutico, honorários, início e término dos trabalhos, e a questão da transferência como mola propulsora desse trabalho, visando uma reconstrução da própria existência (Lins, et al., 2009).

${ }^{1}$ Utiliza-se por convenção, no campo da saúde mental, a denominação Acompanhamento Terapêutico (AT) - com letra maiúscula — para designar a prática clínica e acompanhante terapêutico (at) - com letra minúscula — quando se tratar do profissional que exerce tal prática. Marcamos o at em itálico, para melhor distingui-lo no texto. 


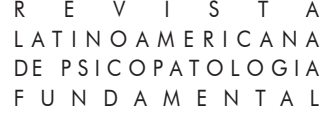

O AT é definido como uma prática itinerante, um tratamento feito em movimento, em que os atendimentos, frutos de uma escuta clínica, se dão tanto em espaços privados como uma residência, um quarto, uma cozinha, quanto em espaços públicos como o consultório, o banco, a igreja etc. Destacamos o nosso olhar para o AT que nessa rede de relações, fica frente à família e ao convívio social do portador, implicando, assim, um corpo a corpo constante, uma disponibilidade para o encontro com outro. Para Palombini (2006) a eficácia dessa prática clínica se dá, sobretudo, naqueles casos ditos não aderirem ao tratamento (convencional).

Importante ressaltar que não há um saber preestabelecido sobre um caso, mas um saber que se constrói, no atendimento. É essa mudança observada por Foucault que introduziu a discussão do caso clínico como mola propulsora do trabalho no hospital. Depois de quase dois séculos a construção do caso no trabalho do AT em associação com a psicanálise pode aí introduzir um corte.

Mas isso não é suficiente para um analista. Ele deve conseguir que esse sujeito que sofre, passe da posição de bela alma, da posição de vítima inocente, para a posição daquele que suspeita de uma cumplicidade própria, mesmo sendo enigmática, no sintoma do qual ele sofre. Somente partindo desse ponto haverá trabalho do sujeito. Essa passagem é fundamental. É uma passagem que não pode ser provocada, que poderá ser explicitada somente se se estiver atento à construção. (Viganò, 1999, p. 43-44)

$\mathrm{Na}$ construção do caso, parte-se do princípio de que a equipe que acompanha um usuário "nada sabe a seu respeito. (...) a autoridade clínica passa a ser o saber do paciente - este é o saber focalizado na construção do caso clínico" (Andrade, 2005, p. 48). Entenda-se por "autoridade clínica", o que Viganò define, justamente na disjunção para com a acepção médica acima historicizada com Foucault: não mais a autoridade clínica a partir do discurso médico, "mas a partir da experiência freudiana, ou seja, a partir da autoridade clínica do próprio paciente, enquanto inventor de seu próprio sintoma" (Viganò, 2000, p. 44). Eis o que tentaremos articular com os exemplos a seguir.

\section{Um exemplo, a partir de um caso do CAPSi João de Barro}

O caso clínico que procuramos construir na sequência, é o de um rapaz atendido através do dispositivo do AT no CAPSi João de Barro, localizado no Rio de Janeiro. Daniel, 19 anos, já não frequentava mais o CAPSi havia cerca 


\section{SAÚDE MENTAL}

de dois anos. Ele pouco saía de casa e era apenas levado para um "centro de reabilitação" por um motorista, diariamente: o AT também é um recurso quando há impossibilidade de acesso à via pública por parte do sujeito, mantido em uma "atmosfera pesada e asfixiante" (Palombini, 2009, p. 300). E foi nessa atmosfera que encontramos Daniel na primeira visita à sua casa: ele andava de um lado a outro, sem conseguir parar, comia vários objetos que via pela frente, e quando falava era para responder uma ou outra pergunta, geralmente repetindo a última palavra pronunciada. Nas primeiras semanas, nos mantivemos na casa do paciente, apesar de sua visível indiferença.

Por vezes algumas palavras soltas eram proferidas de forma bastante rápida, o que tornava difícil a compreensão. Em sua fala e ações, os significantes pareciam dispersos, o que demonstrava faltar ao sujeito um sustentáculo que minimamente pudesse organizar esses significantes. Quinet (2000, p. 88) mostra que, na esquizofrenia, a dispersão dos significantes mestres $\left(\mathrm{S}_{1}\right)$ testemunha a falta de um suporte das representações-meta, o que impede aos pensamentos terem uma meta comandada pelo desejo. Foi então essa a hipótese diagnóstica com a qual iniciamos.

A razão que nos leva a querer compartilhar esse caso aqui, baseia-se no surpreendente efeito que se seguiu à condução de Daniel para fora de sua casa, graças ao dispositivo clínico AT. No momento em que passamos a sair e a caminhar pelas imediações de sua casa, Daniel pareceu mudar radicalmente: seu andar não pareceu mais desnorteado e seu hábito de andar com as mãos dentro das calças subitamente cessou; é como se, na rua, algo o contivesse. Tal efeito já fora apontado por Palombini (2007) quando comentava outra experiência:

o inconsciente a céu aberto da psicose encontra no espaço aberto da rua, em sua acepção de espaço público, uma via privilegiada de expressão. $\mathrm{Na}$ diversidade de seus elementos, ao mesmo tempo em que a rua é propícia à presentificação, para o psicótico, da desmedida de seu Outro, ela é pródiga, também, na oferta de meios com que lhe impor comedimento. Mas além disso, a rua é campo fértil para a extração de materiais com os quais o psicótico pode trabalhar na direção de produzir seu sintoma, na sua amarração singular ao laço social. (p. 158)

O próprio território e a organização social que dentro dele se estabeleceu funcionaram como substratos para que Daniel pudesse estabelecer um afastamento em relação à sua condição autística. Essa possibilidade de resgatar o contato com a realidade confirma os dizeres de Freud em uma bela passagem do "Esboço da psicanálise", onde afirma que o afastamento em relação à realidade na psicose não é absoluto e nem irreversível: 


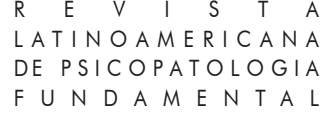

Mesmo num estado tão afastado da realidade do mundo externo como o de uma confusão alienatória, aprende-se com os pacientes, após seu restabelecimento, que, na ocasião, em algum canto da mente (como o dizem) havia uma pessoa normal escondida, a qual, como um espectador desligado, olhava o tumulto da doença passar por ele. (Freud, 1938/2006f, p. 215)

Após alguns meses de atendimento, pudemos verificar essa afirmação na prática, quando Daniel, rompendo bruscamente um longo silêncio, diz: "eu sinto muito, eu sinto prá caçamba!", e novamente silenciou. Em seguida, após repetir ininterruptamente a frase "precisa saber", olhou sua atendente e disse: "Você precisa saber, você precisa saber sobre mim". Evidenciaram-se assim as observações da cisão que operava em Daniel, e que Freud denominou de Spaltung do sujeito, essa que o divide em duas atitudes psíquicas: "uma delas, a normal, que leva em conta a realidade, e outra que, sob a influência das pulsões, desliga o eu da realidade" (Idem).

Mas, fundamentalmente, essa fala de Daniel nos remete ao cerne do que podemos entender cada vez mais desse dispositivo clínico, na acepção que the é dada pela presença do discurso psicanalítico no campo da saúde mental, em que o próprio usuário é o verdadeiro mestre de ensinamentos sobre o saber e sobre a estrutura do inconsciente (Di Ciaccia, 1999, p. 95), permitindo com que, na relação com ele, surja a direção do tratamento a ser dada pelo AT e pela equipe. Se, por um lado, isso responde ao apelo social que a Reforma Psiquiátrica engendrou, permitindo trabalhar de forma articulada, com um "espaço para o exercício de alteridades reciprocamente reconhecidas porque visíveis ou reconhecíveis; (...) provocando movimento, abertura, troca social no cotidiano do paciente que, sob vários aspectos, leva uma vida marcada pela segregação" (Chaui-Berlinck, 2010, p. 92), por outro, no momento em que se passa a levar em conta os ensinamentos e o inconsciente do sujeito, comparece a psicanálise, na sua especificidade.

O que era possível saber? Aos oito anos, Daniel frequentava a escola, já tendo sido, inclusive, alfabetizado. Foi quando sobreveio o primeiro surto e se tornou insuportável continuar na escola. Os pais ainda insistiram, colocando Daniel em uma classe especial, mas a resistência das escolas em recebê-lo e o consequente descrédito dos pais em relação à possibilidade de o filho retornar aos estudos, os conduziu a matriculá-lo em um centro de reabilitação para pessoas com deficiência.

Os centros de reabilitação têm se mostrado uma opção bastante comum nos casos de crianças e adolescentes que entram em surto em idade escolar, o que evidencia o quanto nosso sistema de ensino, público e particular, ainda 


\section{SAÚDE MENTAL}

engatinha em relação à possibilidade de inclusão desses sujeitos com grave sofrimento psíquico. Nos casos de bem menor gravidade, quando a criança simplesmente não se adequa ao controle disciplinar proposto pela escola, busca-se cada vez mais contê-la através da medicalização. Mas o que fazer então com um sujeito que, tomado pela loucura, subverte as leis da instituição escolar a tal ponto que nem mesmo o uso de medicamentos é capaz de contê-lo? Afinal, como combater uma força tão intensa, capaz de desligar o eu do sujeito da realidade e arrastá-lo para longe?

Os chamados centros de reabilitação trazem como objetivo reinserir o indivíduo na sociedade através de um treinamento para adquirir habilidades sociais. Durante a visita à instituição em que Daniel fora matriculado, pudemos verificar que os alunos são orientados a cumprir diversas tarefas, utilizando-se de artifícios oriundos de terapias comportamentais. Para ir ao banheiro, por exemplo, o indivíduo deve colar em um mural um cartão com a frase "quero ir ao banheiro". Cada tarefa cumprida implica a retirada do cartão correspondente do mural. Após o cumprimento de todas as tarefas, tem-se uma atividade livre. É curioso que essa técnica tenha como objetivo adaptar o aluno à sociedade sem que nenhuma questão atrelada ao social seja posta em questão, embora os alunos estejam durante todo o tempo dividindo a sala com os demais. O fato é que a obrigação em se cumprir as tarefas propostas serve de álibi para que os fenômenos oriundos das interações entre os alunos sejam colocados de lado. Um desses fenômenos, por exemplo, ocorreu quando, na instituição em questão, observamos uma sequência de brigas entre dois meninos. A atitude da terapeuta da instituição foi coibir a ação do garoto que iniciara as brigas, sem levar em conta que o outro menino provocava o agressor insistentemente.

Em "Psicologia das massas e análise do eu" (1921/1996), Freud aponta que certos fenômenos acontecem notadamente em situações de grupo e que isso não seria a prova da existência de uma nova pulsão social, mas de que "a pulsão social talvez não seja uma pulsão primitiva, insuscetível de dissociação, e que seja possível descobrir os primórdios de sua evolução num círculo mais estreito, tal como o da família" (p. 82). Donde, não é porque uma instituição dispõe diversos alunos em uma sala, nem tampouco por que os treina para atuarem em situações que exigem habilidades sociais, que ela é uma instituição que trata apenas de questões sociais! Por outro lado, a psicologia individual é "ao mesmo tempo também uma psicologia social" (p. 81).

Assim que ingressamos no caso e passamos a circular no território com Daniel, sua mãe relata um sonho: o filho era levado para cada vez mais longe de casa e ela não conseguia alcançá-lo. Após o relato do sonho, a mãe pôde falar de sua angústia ao vê-lo afastar-se de casa, e a partir daí passou a 


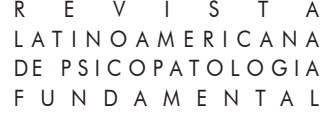

implicar-se cada vez mais no tratamento do filho. Foi surpreendente o quanto Daniel foi pouco a pouco surgindo no discurso como um sujeito, inclusive na fala do pai que disse: "Até me assustei quando ele perguntou se eu iria comer o bife que estava preparando".

Diante dos avanços de Daniel, percebeu-se que seria bastante interessante o seu retorno ao ensino regular. De início, os pais do rapaz se mostraram descrentes em relação a essa possibilidade, julgando que seria impossível para ele permanecer em uma sala de aula. No entanto, confrontada à observação de diversos técnicos do CAPSi, segundo os quais os avanços de Daniel estavam visíveis, a mãe desabafou: "Acho que na verdade só eu e meu marido não acreditamos no nosso filho". Algumas semanas depois, quando novamente questionada, por telefone, se ela procurou realizar a sua matrícula em uma escola, ela decide mudar o trajeto que estava fazendo de carro: "Vou dar meia-volta agora mesmo", indo matriculá-lo, através do EJA (Educação de Jovens e Adultos), na turma regular de uma escola.

Freud (1987/1911, p. 78) ensina que, após o período de catástrofe interna da psicose, a tentativa de restabelecimento do sujeito, por mais bem- -sucedida que seja, jamais se dá inteiramente, pois nem mesmo a ave Fênix, após renascer, 136 é a mesma, embora conserve o nome. Não sabemos por quais caminhos o psicótico poderá seguir depois de uma crise, mas é fundamental que os possamos acompanhar, apostando na incrível capacidade humana de ressurgir das cinzas.

\section{Referências}

Agamben, G. (2005). O que é dispositivo? Outra travessia, 5. Recuperado de https:// periodicos.ufsc.br/index.php/Outra/article/view/12576/11743.

Andrade, R. D. R. (2005, junho). Discussão x construção do caso clínico. Mental, 4. Recuperado de http://pepsic.bvsalud.org/scielo.php?pid=S1679$44272005000100004 \&$ script $=$ sci_arttext.

Araújo, F. (2005, dezembro). Do amigo qualificado à política da amizade. Estilos da Clinica, 19. Recuperado de http://pepsic.bvsalud.org/scielo.php?script=sci arttext\&pid=S1415-71282005000200005\&lng=pt\&tlng=pt.

Bertolote, J. M. (1996). Em busca de uma identidade para a Reforma Psiquiátrica. In A. Pitta (Org.), Reabilitação psicossocial no Brasil (pp. 155-158), São Paulo, SP: Hucitec.

Borges, A. (2010, agosto). Elementos mínimos para a construção do caso clínico na prática entre vários. CliniCAPS, 11. Recuperado de http://www.clinicaps.com.br/ clinicaps_revista_11_art_02.html. 


\section{SAÚDE MENTAL}

Bursztyn, D. C., \& Figueiredo, A. C. (2012, junho). O tratamento do sintoma e a construção do caso na prática coletiva em saúde mental. Tempo psicanalítico, $X L I V(1), 131-145$.

Chaui-Berlinck, L. (2010). O acompanhamento terapêutico e a formação do psicólogo: por uma saúde humanizada. Arquivos Brasileiros de Psicologia, $\operatorname{VXII}(1), 90-96$.

Couto, R. H. O., \& Alberti, S. (2008, dezembro). Contribuição ao debate entre a psicanálise e a atual reforma psiquiátrica brasileira. Mental, VI(11), 15-33.

Di Ciaccia, A. (1999, setembro). Da função de Um à prática feita por muitos. Curinga - Psicanálise e Saúde Mental, 13, 60-65.

Dunker, C. I. L. (2011). Estrutura e constituição da clínica psicanalítica: uma arqueologia das práticas de cura, psicoterapia e tratamento. São Paulo, SP: Annablume.

Elia, L. (2000). Psicanálise: clínica \& pesquisa. In S. Alberti, \& L. Elia (Orgs.), Clínica e pesquisa em psicanálise (pp. 19-35). Rio de Janeiro, RJ: Rios Ambiciosos.

Figueiredo, A. C. (2004, março). A construção do caso clínico: uma contribuição da psicanálise à psicopatologia e à saúde mental. Revista Latinoamericana de Psicopatologia Fundamental, VII(1), 75-86.

Foucault, M (1980). O nascimento da clínica. Rio de Janeiro, RJ: Forense Universitária.

Foucault, M. (1979). Microfisica do poder. Rio de Janeiro, RJ: Graal.

Freud, S. (1987). Notas psicanalíticas sobre um relato autobiográfico de um caso de paranoia (dementia paranoides). In Edição Standard Brasileira das Obras Psicológicas Completas de Sigmund Freud (pp. 13-89). Rio de Janeiro, RJ: Imago. (Trabalho original publicado em 1911).

Freud, S. (1996a). Totem e tabu. In Edição Standard Brasileira das Obras Psicológicas Completas de Sigmund Freud (pp.13-168). Rio de Janeiro, RJ: Imago. (Trabalho original publicado em 1912).

Freud, S. (1996b). (Trabalho original publicado em 1918).

Freud, S. (1996c). Linhas de progresso na terapia psicanalítica. In Edição Standard Brasileira das Obras Psicológicas Completas de Sigmund Freud (pp. 173-181). Rio de Janeiro, RJ: Imago. (Trabalho original publicado em 1919).

Freud, S. (1996d). Psicologia das massas e análise do eu. In Edição Standard Brasileira das Obras Psicológicas Completas de Sigmund Freud (pp. 79-159). Rio de Janeiro, RJ: Imago. (Trabalho original publicado em 1921).

Freud, S. (1996e). O mal-estar na civilização. In Edição Standard Brasileira das Obras Psicológicas Completas de Sigmund Freud (pp. 67-150). Rio de Janeiro, RJ: Imago. (Trabalho original publicado em 1930). 


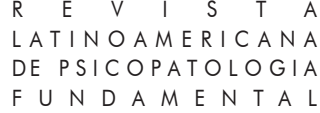

Freud, S. (1996f). Esboço de psicanálise. In Edição Standard Brasileira das Obras Psicológicas Completas de Sigmund Freud (pp. 157-221). Rio de Janeiro, RJ: Imago. (Trabalho original publicado em 1938).

Lacan, J. (2003). Proposição de 9 de outubro de 1967 sobre o psicanalista da Escola. In Outros Escritos (pp. 248-264). Rio de Janeiro, RJ: Zahar.

Lins, C., et al. (2009, fevereiro). Clínica ampliada em saúde mental: cuidar e suposição de saber no acompanhamento terapêutico. Ciência \& Saúde Coletiva, $X I V(1), 205-215$.

Nogueira, L. C. (2004, junho). A pesquisa em psicanálise. Psicol. USP, XV(1-2), 83-106.

Palombini, A. L. (2006, setembro). Acompanhamento terapêutico: dispositivo clínicopolítico. Psychê, $X(18), 115-127$.

Palombini, A. L. (2007). Psicanálise a céu aberto. In Associação Psicanalítica de Porto Alegre - APPOA (Org.), Psicose: aberturas da clínica (pp. 156-175). Porto Alegre, RS: APPOA.

Palombini, A. L. (2009, agosto). Utópicas cidades de nossas andanças: flânerie e amizade no acompanhamento terapêutico. Fractal: Revista de Psicologia, XXI (2), 295-318.

Pitta, A. (1996). O que é a reabilitação psicossocial no Brasil, hoje. In A. Pitta (Org.), Reabilitação Psicossocial no Brasil (pp. 19-26). São Paulo, SP: Hucitec.

Quinet, A. (2000). Teoria e clínica da psicose. Rio de Janeiro: Forense Universitária.

Rinaldi, D. L., \& Bursztyn, D. C. (2008, junho). O desafio da clínica na atenção psicossocial. Arquivos Brasileiros de psicologia, $L X(2)$. Recuperado de http://pepsic.bvsalud.org/scielo.php?script=sci_arttext\&pid=S1809$52672008000200005 \& \operatorname{lng}=\mathrm{pt} \& \mathrm{nrm}=$ iso.

Saraceno, B. (1998). A concepção de reabilitação psicossocial como referência para as intervenções terapêuticas em Saúde Mental. Revista de Terapia Ocupacional da Universidade de São Paulo, IX(1), 26-31.

Val, A. C, \& Lima, M. A. C. (2014, junho). A construção do caso clínico como forma de pesquisa em psicanálise. Ágora: Estudos em Teoria Psicanalítica, XVII(1). Recuperado de https://dx.doi.org/10.1590/S1516-14982014000100007.

Veloso Filho, C. L. (2005). A clínica geral da nova psicanálise: uma contribuição para o campo da saúde coletiva. Dissertação de mestrado, Núcleo de Estudos de Saúde Coletiva no departamento de Medicina Preventiva da Universidade Federal do Rio de Janeiro - UFRJ, Rio de Janeiro, RJ.

Viganó, P. (1999). A construção do caso clínico. Revista Curinga. Escola Brasileira de Psicanálise, 13, 39-48.

Viganó, P. (2000). Comentários clínicos. Opção Lacaniana Revista Brasileira Internacional de Psicanálise, 38, 44-66. 


\section{SAÚDE MENTAL}

Vorcaro, A. (2010). Psicanálise e método científico: o lugar do caso clínico. In F. Kyrillos Neto, \& J. O. Moreira (Orgs.), Pesquisa em psicanálise: uma transmissão na Universidade (pp.11-23). Barbacena, MG: EdUEMG.

\section{Resumos}

(Therapeutic monitoring and psychoanalysis: a small history and clinical case)

We discuss therapentic monitoring based on a brief history of clinical tools used in the mental health field. This discussion is based on transference as a psychoanalytical concept. We hypothesize that, in everyday work carried out outside doctors' offices' models, users' words instigate us to establish a connection between theory and practice - and that psychoanalysis itself can gain from it new knowledge for its clinical practices.

Keywords: Psychoanalysis, clinical tools, therapeutic monitoring, clinical case

(Suivi thérapeutique et psychanalyse: bref historique et cas clinique)

S'étayant sur un bref historique des dispositifs cliniques utilisés dans le domaine de la santé mentale, cet article discute la pratique du suivi thérapeutique. Cette discussion s'instrumentalise du transfert en tant que notion psychanalytique. Il émet les hypothèses que dans ce travail effectué quotidiennement en dehors du domaine des cabinets, la parole d'un utilisateur suggère un lien entre la théorie et la pratique et que la psychanalyse elle-même acquiert ainsi un nouveau savoir faire clinique.

Mots clés: Psychanalyse, dispositif clinique, suivi thérapeutique, cas clinique

(El monitoreo terapéutico y el psicoanálisis: corto historial y caso clínico)

A partir de un breve historial de los dispositivos clínicos utilizados en el campo de la salud mental, se analiza la práctica del monitoreo terapéutico. Esta discusión se instrumentaliza por la transferencia, como concepto psicoanalítico. Surgen entonces las hipótesis de que en ese trabajo realizado diariamente, por fuera del molde de los consultorios, el discurso de un usuario nos instiga a un enlace entre la teoría y la práctica y el propio psicoanálisis adquiere un nuevo saber-hacer clínico.

Palabras clave: Psicoanálisis, dispositivo clínico, monitoreo terapéutico, caso clínico 


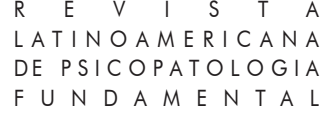

(Therapeutische Begleitung und Psychoanalyse: kurzer historischer Rückblick und klinischer Fall)

Basierend auf einem kurzen historischen Rückblick von klinischen Methoden im Bereich der psychischen Gesundheit, diskutiert der vorliegende Artikel die Praxis der therapeutischen Begleitung. Diese Diskussion leitet sich von der Übertragung ab, in Sinne des psychoanalytischen Konzepts. Wir erstellen die folgenden Hypothesen: 1. In dieser täglichen Arbeit, die außerhalb des Praxismodells stattfindet, unterstützt die Sprache des Benutzers die Verbindung zwischen Theorie und Praxis und 2. die Psychoanalyse kann dadurch neue Erkenntnisse für die klinische Arbeit erwerben.

Schlüsselwörter: Psychoanalyse, klinische Methode, therapeutische Begleitung, klinischer Fall

(跟踪治疗和心理分析：历史和临床病例）

本文回顾了心理健康领域中的治疗方法简史，讨论了跟踪治疗的临床实

践。我们研究了作为精神分析学的工具, “疏导转移” 一词的概念和应用。我 们发现，在诊所之外，在日常运用中，“疏导转移”的涵义不断丰富完善。因 为病人的倾诉，促使专业人员把理论和实践紧密联系在一起，从而促进临床实 践的进一步完善。

关键词: 心理分析, 临床方法, 伴随治疗, 临床病例。

Citação/Citation: Alberti, S., Teixeira, L. C., Beteille, I. M., Rodrigues, S. W. D. M., \& Martinez, C. R. B. S. (2017, março). O Acompanhamento Terapêutico e a psicanálise: pequeno histórico e caso clínico. Revista Latinoamericana de Psicopatologia Fundamental, 20(1), 128-141.

Editores do artigo/Editors: Profa. Dra. Ana Cristina Figueiredo e Profa. Dra. Andréa Maris Campos Guerra.

Recebido/Received: 15.9.2016/ 9.15.2016 Aceito/Accepted: 12.11.2016 / 11.12.2016

Copyright: (C) 2009 Associação Universitária de Pesquisa em Psicopatologia Fundamental/ University Association for Research in Fundamental Psychopathology. Este é um artigo de livre acesso, que permite uso irrestrito, distribuição e reprodução em qualquer meio, desde que o autor e a fonte sejam citados / This is an open-access article, which permits unrestricted use, distribution, and reproduction in any medium, provided the original authors and sources are credited.

Financiamento/Funding: As autoras declaram não terem sido financiadas ou apoiadas / The authors have no support ou finding to report.

Conflito de interesses/Conflict of interest: As autoras declaram que não há conflito de interesses / The authors have no conflict of interest to declare. 


\section{SAÚDE MENTAL}

\section{Sonia Alberti}

Professora Associada do Instituto de Psicologia da Universidade do Estado do Rio de Janeiro - IP/UERJ (Rio de Janeiro, RJ, Br); Pesquisadora do CNPq (Brasília, DF, Br); Membro do Colegiado do Programa de Pós-graduação em Psicanálise do IP/UERJ; Membro da Escola de Psicanálise dos Fóruns do Campo Lacaniano (Rio de Janeiro, RJ, Br); Membo do GT Psicanálise, Política e Clínica da ANPPEP.

Rua João Afonso, 60 casa 22

22261040 Rio de Janeiro, RJ, Br.

sonialberti@gmail.com

\section{Leônia Cavalcante Teixeira}

Professora Titular do Programa de Pós-Graduação em Psicologia da Universidade de Fortaleza - Unifor (Fortaleza, CE, Br); Pesquisadora do CNPq (Brasília, DF, Br); Doutora em Saúde Coletiva com Pós-Doutorado em Psicologia; Ms. em Educação; Psicanalista; Membo do GT Psicanálise, Política e Clínica da ANPPEP.

Rua Henriqueta Galeno, 1080/2301

60135-420 Fortaleza, CE, Br.

leonia.ct@gmail.com

\section{Irene Moura Beteille}

Mestre e Doutoranda no Programa de Pós-graduação em Psicanálise - IP/UERJ (Rio de Janeiro, RJ, Br); Membo do GT Psicanálise, Política e Clínica da ANPPEP.

Rua Izidro de Figueiredo, 24/201 - Tijuca

20271-100 Rio de Janeiro, RJ, Br

irenebeteille@hotmail.com

\section{Sônia Wan Der Maas Rodrigues}

Mestre e Doutoranda em Psicologia pela Universidade de Fortaleza - Unifor (Fortaleza, $\mathrm{CE}, \mathrm{Br}$ ), com pesquisa financiada pela Coordenação de Aperfeiçoamento de Pessoal de Nível Superior; Psicóloga clínica; Membo do GT Psicanálise, Política e Clínica da ANPPEP.

Rua Júnior Rocha, 245 - Parque Manibura

60713-815 Foraleza, CE, Br

soniawandermaas@gmail.com

\section{Carla Renata Braga de Souza Martinez}

Mestre e Doutoranda em Psicologia pela Universidade de Fortaleza - Unifor (Rio de Janeiro, RJ, Br), com pesquisa, financiada pela Coordenação de Aperfeiçoamento de Pessoal de Nível Superior; Psicóloga.

Rua Israel Bezerra, 1057/803 - DionísioTorres

60135-460 Fortaleza, CE, Br

carlarenatabs@gmail.com

This is an open-access article, which permits unrestricted use, distribution, and reproduction in any medium for non-commercial purposes provided the original authors and sources are credited. 